\section{Reply to E. Laffon et al.}

We read with interest the comment by Laffon and Marthan $^{1}$ in response to our recent article in Haematologica "Prognostic value of FDG-PET in patients with mantle cell lymphoma: results from the LyMa-PET Project". ${ }^{2}$ They suggested reporting confidence limits for the measurement of standardized uptake value (SUV) to account for technical factors that lead to measurement uncertainty.

Based on data obtained in patients with lung cancer, ${ }^{3}$ they estimated a measurement error of $13.9 \%$ for SUVmax. Therefore, they suggest that the cutoff value of 10.3 quoted in our study may be completed by a lower and upper limit of 8.8 and 11.7. We acknowledge that SUVmax is affected by measurement errors and we agree with Laffon and Marthan that these should be minimized. Nevertheless, measurement uncertainty cannot be evaluated precisely in our study by the method proposed by Laffon and Marthan. Indeed, these latter evaluated uncertainty on a rather small cohort of patients (only 12 patients) and especially in a mono-centric study using only one positron emission tomography (PET) system without respiratory-gating. ${ }^{4}$ It is not clear, if this kind of conclusion can be transposed to a multi-centric cohort where the uncertainties per system can be very different. Moreover, Laffon and Marthan reported the use of dynamic PET imaging within 60-110 min after injection (1 step, 10 consecutive frames of $2.5 \mathrm{~min}$ each), a time-period where fluorodeoxyglucose (FDG) uptake is subject to significant dynamic. Because consistency of SUV measurements partly depends on the strict observance of the uptake time, a $60 \mathrm{~min}$ interval is recommended with an acceptable range of $55-75$ min. ${ }^{5}$

However, Laffon and Marthan raised an important point which is the error rate inherent to SUVmax measurement. This is well recognized in the field of PET imaging. ${ }^{6,7}$ PET centers need to standardize procedures for patient preparation, image acquisition, data processing, and interpretation to obtain reproducible results. Yet, the LyMa-PET study was initially conducted without an attempt to harmonize local acquisition/reconstruction settings as patients were mainly included within the routine clinical workflow. A few post-acquisition harmonization approaches have been recently published to minimize inter-center variability. ${ }^{8,9} \mathrm{~A}$ recent study of cervical cancer supported the benefits of harmonization in that respect. ${ }^{10}$

Nevertheless, despite all these potential measurement errors, robust significant data were reported in the LyMaPET study. The results remain the same for the SUVpeak and to a lesser extent for the SUVmean. These are all the more notable because they were obtained in "real-life" conditions. As the different SUV metrics showed similar prognostic values, we chose to assess the FDG-uptake only as measured with the SUVmax, this metric being the most widely used. It is noteworthy, that after the first description of a similar score by our team in a retrospective study, ${ }_{11}^{11}$ and again, despite these potential measurement uncertainties, this new prospective study seems to point in the same direction.

Finally, as already notified to Laffon et al. in a previous response to one of their numerous comments, ${ }^{12}$ ensuring reproducibility by establishing clear guidelines is warranted for FDG-PET to be fully endorsed by the hematology community and used in everyday routine in mantle cell lymphoma (MCL) patients. In such guidelines for the development of PET-adapted approaches, a specific cutoff should ideally distinguish between two groups with different risks. Taking a lower and upper limit as proposed by Laffon and Marthan would create a gray zone and does not seem appropriate to further use SUVmax in clinical practice.

Clément Bailly, 1,2 Thomas Carlier, ${ }^{1,2}$

Françoise Kraeber-Bodéré, ${ }^{1,2,3}$ Steven Le Gouill ${ }^{1,4}$ and Caroline Bodet-Milin ${ }^{1,2}$

${ }^{1}$ CRCINA, INSERM, CNRS, Université d'Angers, Université de Nantes; ${ }^{2}$ Department of Nuclear Medicine, CHU Nantes, Nantes; ${ }^{3}$ Department of Nuclear Medicine, ICO-René Gauducheau, SaintHerblain and 'Department of Haematology, CHU Nantes, Nantes, France.

Correspondence: STEVEN LE GOUILL steven.legouill@chu-nantes.fr

\section{doi:10.3324/haematol.2019.240523}

Information on authorship, contributions, and financial \& other disclosures was provided by the authors and is available with the online version of this article at www. haematologica.org.

\section{References}

1. Laffon E, Marthan R. Comment on: "Prognostic value of FDG-PET in patients with mantle cell lymphoma: results from the LyMa-PET Project" Haematologica. 2020;(1):42.

2. Bailly C, Carlier T, Berriolo-Riedinger A, et al. Prognostic value of FDG-PET in patients with mantle cell lymphoma: results from the LyMa-PET Project. Haematologica. 2019. [Epub ahead of print].

3. Laffon E, Lamare F, de Clermont H, Burger IA, Marthan R. Variability of average SUV from several hottest voxels is lower than that of SUVmax and SUVpeak. Eur Radiol. 2014;24(8):1964-1970.

4. Frood R, McDermott G, Scarsbrook A. Respiratory-gated PET/CT for pulmonary lesion characterisation-promises and problems. $\mathrm{Br} \mathrm{J}$ Radiol. 2018;91(1086):20170640.

5. Boellaard R, Delgado-Bolton R, Oyen WJG, et al. FDG PET/CT: EANM procedure guidelines for tumour imaging: version 2.0. Eur J Nucl Med Mol Imaging. 2015;42(2):328-354.

6. Keyes JW. SUV: standard uptake or silly useless value? J Nucl Med. 1995;36(10):1836-1839.

7. Adams MC, Turkington TG, Wilson JM, Wong TZ. A Systematic Review of the Factors Affecting Accuracy of SUV Measurements. AJR Am J Roentgenol. 2010;195(2):310-320.

8. Orlhac F, Boughdad S, Philippe C, et al. A Postreconstruction harmonization method for multicenter radiomic studies in PET. J Nucl Med. 2018;59(8):1321-1328.

9. Chatterjee $\mathrm{A}$, Vallières $\mathrm{M}$, Dohan $\mathrm{A}$, et al. Creating robust predictive radiomic models for data from independent institutions using normalization. IEEE Transactions on Radiation and Plasma Medical Sciences. 2019;3(2):210-215

10. Lucia F, Visvikis D, Vallières $M$, et al. External validation of a combined PET and MRI radiomics model for prediction of recurrence in cervical cancer patients treated with chemoradiotherapy. Eur J Nucl Med Mol Imaging. 2019;46(4):864-877.

11. Bodet-Milin C, Touzeau C, Leux C, et al. Prognostic impact of $18 \mathrm{~F}$ fluoro-deoxyglucose positron emission tomography in untreated mantle cell lymphoma: a retrospective study from the GOELAMS group. Eur J Nucl Med Mol Imaging. 2010;37(9):1633-1642.

12. Meignan M, Cottereau AS, Versari A, et al. Reply to H.J.A. Adams et al and E. Laffon et al. J Clin Oncol. 2017;35(8):920-923. 